City, IA 52242).

COMMENT. Mobius syndrome is a congenital disorder usually limited to a bilateral paralysis of cranial nerves VI and VII, resulting in facial diplegia and impaired abduction of the eyes in lateral gaze. Other cranial nerves, V, IX, and XII are occasionally involved also. In some cases the involvement of the brainstem nuclei, nerves and muscles is more diffuse, leading to an expanded form of the syndrome. Limb and other craniofacial malformations may occur. In the case reported above, the pathology was particularly extensive and the infant died soon after birth. The syndrome has multiple presumed causes. As in the present report, a possible prenatal vascular insuffiency or hypoxic/ischemic event involving embryonic subclavian artery branches may cause gliosis and mineralization of selected cranial nerve nuclei or a more extensive pathology. A dysgenesis of cranial nerve nuclei and hypoplasia of nerves and muscles (oromandibular limb hypogenesis) or a primary myopathy involving facial and external eye muscles are alternative theories,. The timing of the suggested embryonic insult is in the early first trimester, Abuse of benzodiazepines, chorionic villus sampling, and placental bleeding have been invoked as causes. The term Mobius sequence is sometimes preferred to syndrome, better describing a cascade of events secondary to a proposed embryonic insult. Mobius sequence is preferred by the authors of the following report.

\title{
MOBIUS SYNDROME AND AUTISTIC SPECTRUM DISORDER
}

The relation between autistic spectrum disorder and Mobius syndrome was studied in 25 patients ( 18 males and 7 females) included in a multidisciplinary study of Mobius sequence at the Department of Child and Adolescent Psychiatry, Goteborg University, Sweden. Autistic spectrum disorder and learning disability were present in more than one third of patients. The findings point to a possible brainstem insult in early pregnancy as a cause in some cases of autism. (Johansson M, Wentz E, Fernell E et al. Autistic spectrum disorders in Mobius sequence: a comprehensive study of 25 individuals. Dev Med Child Neurol May 2001;43:338-345). (Respond: Maria Johansson MD, Department of Child and Adolescent Psychiatry, Gotegorg University, Kungsgatan 12, SE-411 19 Goteborg, Sweden).

COMMENT. Brainstem dysfunction has been proposed as a possible mechanism of autism (Gillberg et al, 1983). The present study offers some support for this hypothesis.

\section{JUVENILE DERMATOMYOSITIS WITH GENERALIZED EDEMA}

A 7-year-old girl presenting with an 8-week history of fatigue, myalgia, dyspnea, and generalized, nonpitting edema of the extremities, face, chest, and abdomen, is reported from the Walter Reed Army Medical Center, Washington, DC. A heliotrope and malar rash had developed, and muscles were tender to palpation. A severe proximal muscle weakness, elevated serum muscle enzymes, and heliotrope rash were characteristic of juvenile dermatomyositis (JDM). Other diseases characteristic of generalized anasarca, such as nephrosis, liver disease, hypothyroidism, and malignancy, were excluded. Seventeen additional cases of JDM and anasarca were cited in the literature. Response to oral steroids was poor, and high dose long duration IV therapy was needed. (Mitchell JP, Dennis GJ, Rider LG. Juvenile dermatomyositis presenting with anasarca: a possible indicator of severe disease activity. I Pediatr June 2001;138:942-945). (Reprints: Jeanne P Mitchell $\mathrm{MD}$, Department of Rheumatology and Clinical Immunology, Walter Reed Army Medical Center, 6900 Georgia Ave NW, Ward 77, Washington, DC 20307). 